\title{
International Spinal Cord Injury Physical Therapy-Occupational Therapy Basic Data Set (Version 1.2)
}

\author{
Kim D. Anderson $\mathbb{D}^{1,2} \cdot$ Edelle C. Field-Fote ${ }^{3,4,5} \cdot$ Fin Biering-Sørensen $\mathbb{D}^{6} \cdot$ Anne Bryden $^{7} \cdot$ Lisa A. Harvey ${ }^{8} \cdot$ \\ Linda Jones $^{9}$ - M. J. Mulcahey ${ }^{10}$. Sara Mulroy ${ }^{11,12}$ • Vanessa K. Noonan ${ }^{13,14}$ - Marcel W. M. Post $\mathbb{D}^{15}$. \\ Rüdiger Rupp $\mathbb{1}^{16} \cdot$ Mary Schmidt-Read ${ }^{17}$
}

Received: 2 June 2020 / Revised: 31 July 2020 / Accepted: 3 August 2020

(c) The Author(s), under exclusive licence to International Spinal Cord Society 2020

\begin{abstract}
Study design Expert workgroup consensus, focused literature review, and vetting via feedback from international presentations and spinal cord professional membership groups.

Objectives Develop and refine a basic dataset to enable standardized documentation of physical therapy (PT) and occupational therapy (OT) interventions delivered in a controlled clinical trial intended to improve voluntary motor function.

Setting International Expert Working Group.

Methods An international working group with expertise in spinal cord injury, PT, OT, and measurement developed a draft of the International Spinal Cord Injury (ISCI) Physical Therapy-Occupational Therapy (PT-OT) Basic Data Set (BDS). Emphasis was placed on efficiency and practicality of use. The BDS was iteratively refined based on applicable literature, and feedback collected from presentations at the 2017 and 2019 International Spinal Cord Society meetings.

Results The ISCI PT-OT BDS contains seven broad categories of interventions: bed/seated mobility, standing activities, walking/stairs, gross motor upper extremity, fine motor upper extremity, strength training, and endurance training. The first five categories are classified as activity-directed and the last two as impairment-directed interventions. Time spent on interventions per category is recorded in 15-min intervals.

Conclusions The ISCI PT-OT BDS enables standardized documentation of PT-OT activity-directed or impairment-directed interventions. The ISCI PT-OT BDS is a documentation tool to facilitate evaluation of the influence of rehabilitation therapies on motor function in clinical trials of biologic or pharmacologic agents or rehabilitation technologies that are delivered in the clinical setting.
\end{abstract}

\section{Introduction}

Many spinal cord injury (SCI) clinical trials of biologic or pharmacologic agents or rehabilitation technologies target primary endpoints of reduced neurological impairment, as

These authors contributed equally: Kim D. Anderson, Edelle C. Field-Fote

Supplementary information The online version of this article (https:// doi.org/10.1038/s41394-020-00323-z) contains supplementary material, which is available to authorized users.

Kim D. Anderson

kxa304@case.edu

$\triangle$ Edelle C. Field-Fote

Edee.Field-Fote@shepherd.org

Extended author information available on the last page of the article measured by the International Standards for Neurological Classification of SCI (ISNCSCI) [1], and/or functional improvement as measured by the Spinal Cord Independence Measure (SCIM III) [2]. These endpoints are also primary goals of rehabilitation therapies delivered by physical therapists (PTs) and occupational therapists (OTs). In animal models of SCI, there is growing evidence that physical activity, practice, and training influence neural plasticity, neuroprotection, regeneration, neural organization, and neuronal properties [3-6]. In human SCI clinical trials, experimental interventions incorporating physical rehabilitation have the strongest evidence for efficacy [7].

Clinical trialists therefore need to account for the influence of therapies delivered by PTs and OTs when delivered concurrently or as part of experimental interventions or usual care. To accomplish this, systematic documentation of such therapies are needed. There are several comprehensive rehabilitation 
Table 1 Initial matching and ranking results of like interventions in SCIInterventions Classification System (SCI-ICS) and the SCIRehab taxonomies.

\begin{tabular}{|c|c|c|}
\hline SCI-ICS & SCIRehab therapies form & Ranks \\
\hline 11. Muscle power & 12. Strengthening & 1 \\
\hline 25. Walking & 7. Pre gait; 8. Gait & 2 \\
\hline 21. Arm and hand use & 2. Self-feeding & 3 \\
\hline 24. Standing & 6. Upright activities & 4 \\
\hline 31. Moving around inside & $\begin{array}{l}\text { 4. Wheelchair mobility-manual; } 5 \text {. Wheelchair mobility- } \\
\text { power; 7. Pre gait; } 8 \text {. Gait }\end{array}$ & 4 \\
\hline 22. Positions and movements & 2. Bed mobility; 1. Balance & 6 \\
\hline 32. Moving around outside & $\begin{array}{l}\text { 4. Wheelchair mobility-manual; 5. Wheelchair mobility- } \\
\text { power; 7. Pre gait; } 8 \text {. Gait }\end{array}$ & 7 \\
\hline 23. Transfers & 3. Transfers & 8 \\
\hline 13. Muscle tone & 9. Range-of-motion (ROM)/stretching & 9 \\
\hline 33. Washing oneself & 22. Bathing & 10 \\
\hline 15. Sensory functions & 31. Other therapeutic activities & 11 \\
\hline 17. Musculoskeletal pain & $\begin{array}{l}\text { 13. Musculoskeletal treatments/modalities; } 32 \text {. Modalities; } 51 \text {. } \\
\text { Education not covered by other activities }\end{array}$ & 12 \\
\hline 12. Muscle length & 9. ROMIstretching & 13 \\
\hline 14. Joint mobility & 9. ROMIstretching & 13 \\
\hline 36. Dressing & 23. Dressing-lower body; 24. Dressing-upper body & 13 \\
\hline 16. Neuropathic pain & $\begin{array}{l}\text { 44. Psychotherapeutic intervention; } 51 \text {. Education not covered } \\
\text { by other activities covered by other activities; } 54 \text {. } \\
\text { Psychosocial support }\end{array}$ & 16 \\
\hline \multirow[t]{2}{*}{ 19. Cardiovascular system } & 11. Endurance & 16 \\
\hline & 13. Musculoskeletal treatments/modalities & 18 \\
\hline $\begin{array}{l}\text { 26. Handrim wheelchair/(bi) } \\
\text { cycle }\end{array}$ & 4. Wheelchair mobility—manual & 19 \\
\hline 28. Swimming & 19. Aquatic exercises/hydrotherapy & 20 \\
\hline 34. Caring for body parts & 21. Grooming & 20 \\
\hline
\end{tabular}

The bold numbers are the top 10 ranked pairings of interventions of the 21 pairings presented. taxonomies available, including the SCI-Interventions Classification System (SCI-ICS) [8-10], the SCIRehab taxonomy [11-13], and the Rehabilitation Treatment Specification System [14]. However, these extensive taxonomies were developed expressly to investigate the influence of various physical rehabilitation interventions on a wide range of outcomes, and the time required to record the information presents a barrier for their implementation [15-17].

Our working group (composed of international experts in SCI, PT, OT, and measurement) was appointed by the International Spinal Cord Society (ISCoS) Dataset Committee to develop an International Spinal Cord Injury (ISCI) Physical Therapy-Occupational Therapy (PT-OT) Basic Data Set (BDS) as a means to standardize the efficient collection and reporting of the basic content and time of PTOT sessions that are delivered in interventional studies aimed at improving motor function. We developed the ISCI PT-OT BDS in accordance with the guidelines of the ISCI Data Sets [18]. The tool is intended to be used by therapists to indicate the amount of time expended in each category of intervention during each skilled therapy session (individual or group) in an inpatient or outpatient rehabilitation setting.
Data captured with this tool may be useful not only when investigating the effect of experimental interventions, but also when reporting clinical outcomes in case studies, series, and pragmatic trials as well as when evaluating clinical outcomes and/or quality improvement of usual care.

\section{Methods}

We reviewed the two most comprehensive SCI rehabilitation intervention taxonomies available at the time that our working group was formed (the SCI-ICS and SCIRehab taxonomies) to identify PT and OT interventions that might be suitable for inclusion in the dataset. We matched similar interventions between the two taxonomies, resulting in 21 intervention pairings (Table 1). To reach consensus, each member independently reviewed the pairings of interventions and selected those believed to directly influence the ISNCSCI motor and sensory scores and then rank these pairings of interventions in order of importance. There was $100 \%$ agreement that muscle power/strengthening interventions were the most likely to have a direct influence on the ISNCSCI. 
After the matched and ranked pairings of interventions were identified (bold numbers in Table 1), we considered ways to minimize the time and effort required to record the ISCI PT-OT BDS, and opted to:

(1) Focus on categories of interventions rather than individual interventions (e.g., all interventions focused on walking were combined in a single category, rather than separate categories for overground and treadmill locomotor training),

(2) Include only categories of interventions that may potentially influence ISNCSCI motor and sensory scores, and

(3) Cluster similar interventions together into activity- or impairment-directed intervention groupings as defined by the International Classification of Functioning, Disability, and Health.

This process resulted in eleven categories of interventions (Table 2, left), which we further refined using an iterative consensus process to arrive at seven broad categories (Table 2, right), after removal of the following:

(1) Toileting (removed from fine motor upper extremity). This was justified on the basis that data from SCIRehab study indicate that OTs spend very little rehab time on toileting skills (primarily performed by nursing) [17];

(2) Range-of-motion (ROM) activities. This was justified on evidence indicating PT and OT interventions do not have a meaningful lasting effect on joint ROM [19];

(3) Therapeutic activities to normalize sensation. This was justified on the basis that it is unlikely that the ISNCSCI sensory scores, or a functional outcome measure would be improved by these interventions.

Finally, we acknowledged that dose of an intervention is an important consideration and could influence outcomes. We agreed that dose, as defined in terms of time and intensity (physiologic or perceived), also should be captured. However, we concluded that measuring physiologic intensity was not feasible as part of usual care, as this requires specialized equipment, training, and extensive time. We determined that the component of dose related to time was best captured by recording the amount of time spent in each intervention category during a single PT and lor OT session, apportioned into 15-min intervals based on typical reporting in clinical practice. A "simple" means of documenting perceived intensity was much more challenging. We initially selected the Borg scale as a proxy of perceived intensity and used the modified $0-10$ for rating of perceived exertion (RPE) [21].

An initial round of face and content validity testing was conducted before presenting the dataset to a larger audience (described next). We distributed the dataset to PTs and OTs at institutions where several members of our group hold positions. The PTs and OTs participating in the validity assessment were naive to the dataset; we asked them to each only complete the form once, while working with a single patient in a single therapy session. We asked for their comments on the following questions:

(1) Was the intended use of the form clear?

(2) Were the instructions about how to complete the form clear?

Table 2 Collapsing of therapy modalities into categories of interventions.

Initial collapsed categories

1. Strength training

2. Upright position and mobility: walking, standing, transfers with weight bearing through feet

3. Gross motor upper extremity: dressing, washing

4. Fine motor upper extremity: grooming, toileting, self-feeding

5. Wheelchair mobility: inside, outside

6. Positions and movements: balance, bed mobility, changing position

7. Transfers

8. Therapeutic activities to influence sensory function: stimulation, sensory input (rubbing)

9. Stretching, passive movement

10. Endurance training

11. Electrical stimulation (may be used for different purposes, so needs its own category)
Revised collapsed categories

1. Strength training (including electrical stimulation for strength)

2. Endurance training (including electrical stimulation for endurance)

3. Bed/seated control activities: balance, seated transfers, bed mobility

4. Standing control activities: standing, balance, standing transfers with weight bearing through feet

5. Walking, stairs (inside, outside)

6. Gross motor upper extremity: dressing, washing, manual wheelchair mobility

7. Fine motor upper extremity: grooming, self-feeding, buttoning, zipping, adjustment of clothing 
(3) Were the treatment time intervals appropriate?

(4) How long did it take to complete the form?

(5) Was the form easy to use?

(6) Any other suggestions?

Fifteen therapists (nine PTs, six OTs) from four different centers completed the form and provided feedback. Their collective feedback was that the form was easy to interpret and complete; no major revisions of the form were recommended, but helpful comments were provided to improve the instructions.

We presented the dataset at the 2017 ISCoS annual meeting during the workshop "Why (and How to) Report Rehabilitation Activities in Clinical Trials?" for broader input from the SCI rehabilitation and research communities. The two major discussions at the workshop centered around: (1) the exclusive focus on interventions for which there was evidence of an influence on motor function as measured by the ISNCSCI exam, and not including interventions believed to directly influence functional measures such as the FIM Instrument [20] or the SCIM III, and (2) the lack of evidence for the RPE (Borg scale) as an indicator of intensity. First, based on this feedback, the working group reconsidered the focus on ISNCSCI. We concurred that as some SCI clinical trials use the FIM Instrument/SCIM III as an endpoint [22], the intervention categories included in the BDS should encompass those having the potential to influence the FIM Instrument/SCIM III items reflecting voluntary motor function. Intervention categories that had been removed during earlier iterations of the BDS were reevaluated (see Tables 1 and 2). Given that most items on the FIM Instrument/SCIM III are dependent on voluntary motor function (which is captured by the ISNCSCI), and the focus of this BDS is on PT-OT interventions, it was determined that none of the intervention categories that had been removed had potential to influence the FIM Instrument/SCIM III. Second, the working group decided to remove RPE as a proxy for perceived intensity from the dataset.

The revised version of the ISCI PT-OT BDS was presented at the 2019 ISCoS annual meeting during the workshop "The International Spinal Cord Injury Physical Therapy - Occupational Therapy Basic Data Set: Rationale, Evidence, and Vetting." The main discussion at this workshop centered on comments from participants about the need to capture intensity. As our working group had already discussed this issue at length, and could identify no single appropriate measure of intensity, we decided to include the recommendation that the issue of intensity be considered in future revisions of the $\mathrm{BDS}$, at which time a relevant measure may be available. The final version of the ISCI PT-OT BDS (version 1.2) that has been approved through the dataset approval process and slightly revised during review of this publication is provided in the Supplementary Material.

\section{Results}

The final version of the ISCI PT-OT BDS is described below, as well as the rationale for inclusion of each variable. Applicable citations are included from studies wherein effects of the intervention on ISNCSCI or FIM Instrument/ SCIM III were identified. Where no citations are provided, the inclusion of a data variable was based on working group consensus derived from knowledge of studies that used outcome measures other than the ISNCSCI or FIM Instrument/SCIM III as measures of strength and physical functioning.

\section{Identifying information (center name, therapist name, patient identification, date, time)}

The identifying information variables are intended to distinguish the record based on the center, therapist, and patient identification. Date of data collection captures the date of data collection in order to identify data collected in relation to other data collected on the same patient at various time points, as the recording of data on PT and OT rehabilitation interventions may be done at any time after SCI. Time of data collection captures the time of day when the intervention session was delivered in order to distinguish it from data collected during other sessions on the same day. One form is required per session.

\section{Setting}

This variable captures the setting in which the intervention session was delivered. Delivery of rehabilitation interventions may have a different focus depending on the setting. Therefore, identification of either inpatient or outpatient setting is included.

\section{Time (in min)}

For each of the intervention categories described below, time spent on each is recorded in the following $15 \mathrm{~min}$ intervals: $<15,15-29,30-44,45-60$, and $>60$.

\section{Activity-directed interventions-bed/seated activities: balance, seated transfers, and bed mobility}

Sitting balance and seated transfers include interventions delivered while the patient is in an upright seated position, with or without assistive devices, such as a transfer board, and with any amount of physical assistance. Bed mobility includes interventions delivered while the patient is in a lying or sitting position, with or without the use of assistive devices, and with any amount of physical assistance. 
Examples include sitting balance training on even or uneven surfaces as well as seated transfers from bed to wheelchair, wheelchair to toilet or shower, and wheelchair to floor. Bed mobility examples include rolling from supine to side-lying and side-lying to sitting.

\section{Activity-directed interventions-standing activities: standing, balance, and standing transfers weight bearing}

Included are any interventions performed in an upright position, with or without assistive devices or lower extremity bracing, with or without physical assistance (ranging from supervision to maximal assistance), and any number of personnel. This encompasses any intervention that is not considered walking but is performed in a lower extremity weight bearing position. Examples include weight shifting, balance perturbations, stepping up and down (therapeutically, not as stair climbing), sit to and from stand, standing unsupported or supported, standing transfers, and single or double leg stance. Balance is a prerequisite to participating in activities of daily living, as captured by FIM Instrument/SCIM III [23].

\section{Activity-directed interventions-walking and stairs (inside, outside)}

This variable captures any intervention involving more than one active sequential step, which is unrelated to balance or standing training. These interventions would be performed in an upright, lower extremity weight bearing position, with any amount of physical assistance, assistive device(s), or lower extremity bracing. Examples include overground walking, walking over uneven terrain, treadmill gait training, walking inside or outside, and stair climbing with or without use of railings. Interventions may occur in a variety of gait patterns (such as reciprocal, step to, swing through, etc.). Locomotor training is associated with improvements in the ISNCSCI lower extremity motor score [24-26]. In one RCT that included multiple interventions, wherein locomotor training was a large component of the training, improvements in ISNCSCI lower extremity motor score were associated with training [27]. Walking and stair climbing both are encompassed within the FIM Instrument /SCIM III, thus these interventions directed at these activities may influence FIM Instrument /SCIM III scores in the mobility subscales.

\section{Activity-directed interventions-gross motor upper extremity dressing, washing, bathing, and manual wheelchair propulsion}

This variable captures any interventions that incorporate broad movements of the upper extremity at the shoulder and elbow. These interventions involve active movement such as reaching in multiple planes, and include activities performed with physical assistance or adaptive equipment as needed. Activity-directed interventions involving gross motor upper extremity movements have been associated with improvements in functional activity performance [28-30]. Examples include upper body dressing and bathing, wheelchair propulsion, and interventions that incorporate unilateral and bilateral functional reach.

\section{Activity-directed interventions-fine motor upper extremity: grooming, self-feeding, buttoning, zipping, and adjustment of clothing}

This variable captures any interventions intended to facilitate routine daily activities that require active movement to accomplish a fine motor task. These interventions include unilateral, bilateral, and bimanual movements incorporating the elbow, forearm, wrist, and hand. Activities may be performed with or without adaptive equipment or adaptations. Activity-directed interventions involving fine motor upper extremity movements have been associated with improvements in functional activity performance [28-30]. Examples include personal self-care such as grooming, feeding, and clothing management (buttoning, zipping, adjustments). This category also includes office and communication activities such as writing, managing electronic devices (phone, laptop, tablet), as well as leisure/hobby activities (crafts, games), when included in the rehabilitation PT-OT activities. Physical assistance and adaptive equipment may be used as needed.

\section{Impairment-directed interventions-strength training and/or electrical stimulation administered to increase strength}

This variable captures strength training with or without electrical stimulation, which may increase voluntary strength [31, 32]. Therefore, it may affect the ISNCSCI motor scores, which, in turn, could determine the ISNCSCI motor levels and neurological level, and American Spinal Injury Association (ASIA) Impairment Scale (AIS) grade, and FIM Instrument/SCIM III subscores [33]. This variable does not capture electrical stimulation used for purposes other than to increase voluntary strength (e.g., it does not capture electrical stimulation when used as a neuroprosthesis, electrical stimulation used to induce hypertrophy in fully paralyzed muscles, electrical stimulation used to improve cough, electrical stimulation used to increase cardiovascular fitness, electrical stimulation used to decrease spasticity). 


\section{Impairment-directed interventions-endurance training and/or electrical stimulation administered to increase endurance}

This variable captures endurance training with or without electrical stimulation provided with the aim of increasing cardiovascular fitness and/or reducing neuromuscular fatigue. Endurance training (with or without electrical stimulation) may increase voluntary strength. Therefore, it may affect the ISNCSCI motor scores which, in turn, could determine the motor levels, the single neurological level, and AIS grade, and potentially FIM/SCIM III subscores. This variable does not capture electrical stimulation used for purposes other than to increase endurance (e.g., it does not capture electrical stimulation when used as a neuroprosthesis, electrical stimulation used to induce hypertrophy in fully paralyzed muscles, electrical stimulation used to improve cough, electrical stimulation used to increase voluntary strength, electrical stimulation used to decrease spasticity).

\section{Total activity- and impairment-directed intervention time}

This variable indicates the total time spent in the specified session on the activity- and impairment-directed interventions excluding time spent on other activities such as passive transfers, time donning and doffing assistive devices, instruction time, etc. The total time spent is recorded by selecting the corresponding time interval: $<15,15-29$, $30-44,45-60$, and $>60$.

\section{Discussion}

The purpose of the ISCI PT-OT BDS is to standardize the recording of skilled PT and OT interventions that may influence scores on outcome measures that are commonly used in clinical trials. It is not intended to prescribe or compare interventions or guide practice, nor is it intended to be used for billing purposes. Sponsors of clinical trials could utilize this standardized reporting tool to indicate the amount of time spent in each treatment category during a single PT and/or OT therapy session (individual or group; inpatient or outpatient setting), so that the cumulative time spent in these rehabilitation therapies over the course of a clinical trial can be tracked. To reduce reporting burden, the dataset was purposefully kept brief, and time is denoted as a range of minutes. The ISCI PT-OT BDS should be used in conjunction with the ISCI Core Data Set version 2.0 [34].

The working group acknowledges that intensity (both physiologic and perceived) is an essential component of therapy dose, and not capturing it in the dataset is a limitation. As described in "Methods", attempts were made to include a measure of perceived intensity; however, there is no measure that is known to be a valid indicator of intensity for use across all seven categories of interventions. For example, while the Borg RPE is valid for endurance activities, it is not valid for fine motor training activities. Users of the ISCI PTOT BDS have the option to add sensitivity by recording the actual number of minutes, record/count repetitions, use the Borg scale when appropriate, measure oxygen uptake or heart rate, or use activity monitors.

\section{Plans for future development}

We plan to assess inter-rater reliability of the ISCI PT-OT BDS via an international study of PTs and OTs who work with individuals with SCI. In addition, an electronic version of the ISCI PT-OT BDS has been developed using the Android operating system that will be made publicly available. In accord with data protection regulations, the recorded data will be stored directly on the device as opposed to being uploaded to the cloud. Finally, with the ongoing development of sensor-based tracking technologies, it may be possible to incorporate these technologies into the ISCI PT-OT BDS. Using these technologies, it may be possible to incorporate an algorithm to measure intensity, as well as other aspects of activity- and impairment-directed PT-OT interventions.

\section{Conclusion}

The ISCI PT-OT BDS is a tool to capture the content and time of activity-directed and impairment-directed PT and OT interventions administered in an inpatient or outpatient setting. This standardized reporting tool is intended for use while an individual with SCI is receiving PT and/or OT in a controlled clinical trial wherein the goal of the experimental intervention is to improve strength and/or motor function. Use of tool imposes minimal additional burden on the therapist, and has the potential to disentangle the improvements associated with usual care versus those associated with the experimental intervention.

Acknowledgements The authors gratefully acknowledge the contributions of all individuals who provided feedback as part of the workshops and public review process.

\section{Compliance with ethical standards}

Conflict of interest The authors declare that they have no conflict of interest.

Publisher's note Springer Nature remains neutral with regard to jurisdictional claims in published maps and institutional affiliations. 


\section{References}

1. Betz R, Biering-Sørensen F, Burns SP, Donovan W, Graves DE, Guest J. ASIA and ISCoS International Standards Committee et al. The 2019 revision of the International Standards for Neurological Classification of Spinal Cord Injury (ISNCSCI)-what's new?. Spinal Cord. 2019;57:815-7.

2. Catz A, Itzkovich M, Tesio L, Biering-Sorensen F, Weeks C, Laramee MT, et al. A Multicenter International Study on the Spinal Cord Independence Measure, Version III: Rasch psychometric validation. Spinal Cord. 2007;45:275-91.

3. Fouad K, Tetzlaff W. Rehabilitative training and plasticity following spinal cord injury. Exp Neurol. 2012;235:91-99.

4. Sandrow-Feinberg HR, Houle JD. Exercise after spinal cord injury as an agent for neuroprotection, regeneration, and rehabilitation. Brain Res. 2015;1619:12-21.

5. Torres-Espín a, Forero J, Fenrich KK, Lucas-Osma AM, Krajacic A, Schmidt A, et al. Eliciting inflammation enables successful rehabilitative training in chronic spinal cord injury. Brain. 2018;141:1946-62.

6. García-Alías G, Barkhuysen S, Buckle M, Fawcett JW. Chondroitinase $\mathrm{ABC}$ treatment opens a window of opportunity for taskspecific rehabilitation. Nat Neurosci. 2009;12:1145-51.

7. Gomes-Osman J, Cortes M, Guest J, Pscual-Leone A. A systematic review of experimental strategies aimed at improving motor function after acute and chronic spinal cord injury. J Neurotrauma. 2016;33:425-38.

8. van Langeveld SA, Post MW, van Asbeck FW, Postma K, Ten Dam D, Pons K. Development of a classification of physical, occupational, and sports therapy interventions to document mobility and self-care in spinal cord injury rehabilitation. J Neurol Phys Ther. 2008a;32:2-7.

9. van Langeveld SA, Post MW, van Asbeck FW, Postma K, Leenders J, Pons K. Feasibility of a classification system for physical therapy, occupational therapy, and sports therapy interventions for mobility and self-care in spinal cord injury rehabilitation. Arch Phys Med Rehabil. 2008b;89:1454-9.

10. van Langeveld SA, Post MW, van Asbeck FW, ter Horst P, Leenders J, Postma K, et al. Reliability of a new classification system for mobility and self-care in spinal cord injury rehabilitation: the Spinal Cord Injury-Interventions Classification System. Arch Phys Med Rehabil. 2009;90:1229-36.

11. Whiteneck G, Gassaway J, Dijkers M, Jha A. New approach to study the content and outcomes of spinal cord injury rehabilitation: the SCIRehab Project. J Spinal Cord Med. 2009;32:251-9.

12. Natale A, Taylor S, LaBarbera J, Bensimon L, McDowell S, Mumma SL, et al. SCIRehab Project series: the physical therapy taxonomy. J Spinal Cord Med. 2009;32:270-82.

13. Ozelie R, Sipple C, Foy T, Cantoni K, Kellogg K, Lookingbill J, et al. SCIRehab Project series: the occupational therapy taxonomy. J Spinal Cord Med. 2009;32:283-97.

14. Zanca JM, Turkstra LS, Chen C, Packel A, Ferraro M, Hart T, et al. Advancing rehabilitation practice through improved specification of interventions. Arch Phys Med Rehabil. 2019;100:164-71.

15. Franz M, Richner L, Wirz M, von Reumont A, Bergner U, Herzog $\mathrm{T}$, et al. Physical therapy is targeted and adjusted over time for the rehabilitation of locomotor function in acute spinal cord injury interventions in physical and sports therapy. Spinal Cord. 2018;56:158-67.

16. Teeter L, Gassaway J, Taylor S, LaBarbera J, McDowell S, Backus D, et al. Relationship of physical therapy inpatient rehabilitation interventions and patient characteristics to outcomes following spinal cord injury: the SCIRehab project. J Spinal Cord Med. 2012;35:503-26.
17. Ozelie R, Gassaway J, Buchman E, Thimmaiah D, Heisler L, Cantoni K, et al. Relationship of occupational therapy inpatient rehabilitation interventions and patient characteristics to outcomes following spinal cord injury: the SCIRehab project. J Spinal Cord Med. 2012;35:527-46.

18. Biering-Sørensen F, Charlifue S, DeVivo M, Noonan V, Post M, Stripling T, et al. International spinal cord injury data sets. Spinal Cord. 2006;44:530-4.

19. Harvey LA, Katalinic OM, Herbert RD, Moseley AM, Lannin NA, Schurr K. Stretch for the treatment and prevention of contracture: an abridged republication of a Cochrane Systematic Review. J Physiother. 2017;63:67-75.

20. Guide for the uniform data set for medical rehabilitation (including the FIM(TM) instrument). Version 5.1. Buffalo: State University of New York at Buffalo; 1997.

21. Borg E, Kaijser L. A comparison between three rating scales for perceived exertion and two different work tests. Scand J Med Sci Sports. 2006;16:57-69.

22. Badhiwala JH, Ahuja CS, Fehlings MG. Time is spine: a review of translational advances in spinal cord injury. J Neurosurg Spine. 2018;30:1-18.

23. Wirz M, van Hedel HJA. Balance, gait, and falls in spinal cord injury. Handb Clin Neurol. 2018;159:367-84.

24. Alcobendas-Maestro M, Esclarín-Ruiz A, Casado-López RM, Muñoz-González A, Pérez-Mateos G, González-Valdizán E, et al. Lokomat robotic-assisted versus overground training within 3 to 6 Months of incomplete spinal cord lesion: randomized controlled trial. Neurorehabil Neural Repair. 2012;26:1058-63.

25. Hornby TG, Zemon DH, Campbell D. Robotic-assisted, bodyweight-supported treadmill training in individuals following motor incomplete spinal cord injury. Phys Ther. 2005;85:52-66.

26. Buehner JJ, Forrest GF, Schmidt-Read M, White S, Tansey K, Basso DM. Relationship between ASIA examination and functional outcomes in the NeuroRecovery Network Locomotor Training Program. Arch Phys Med Rehabil. 2012;93:1530-40.

27. Jones ML, Evans N, Tefertiller C, Backus D, Sweatman M, Tansey K, et al. Activity-based therapy for recovery of walking in individuals with chronic spinal cord injury: results from a randomized clinical trial. Arch Phys Med Rehabil. 2014;95:2239-.e2.

28. Schönherr MC, Groothoff JW, Mulder GA, Eisma WH. Functional outcome of patients with spinal cord injury: rehabilitation outcome study. Clin Rehabil. 1999;13:457-63.

29. Spooren AIF, Janssen-potten YJM, Snoek GJ, Ijzerman MJ, Kerckhofs E, Seelen HAM. Rehabilitation outcome of upper extremity skilled performance in persons with cervical spinal cord injuries. J Rehabil Med. 2008;40:637-44.

30. Yarkony GM. Benefits of rehabilitation for traumatic spinal cord injury: multivariate analysis in 711 patients. Arch Neurol. 1987;44:93-6.

31. Aravind N, Harvey LA, Glinsky JV. Physiotherapy interventions for increasing muscle strength in people with spinal cord injuries: a systematic review. Spinal Cord. 2019;57:449-60.

32. Harvey LA, Glinsky JV, Bowden JL. The effectiveness of 22 commonly administered physiotherapy interventions for people with spinal cord injury: a systematic review. Spinal Cord. 2016;54:914-23.

33. Stone WJ, Stevens SL, Fuller DK, Caputo JL. Ambulation and physical function after eccentric resistance training in adults with incomplete spinal cord injury: a feasibility study. J Spinal Cord Med. 2019;42:526-33.

34. Biering-Sørensen F, DeVivo MJ, Charlifue S, Chen Y, New PW, Noonan V, et al. International Spinal Cord Injury Core Data Set (version 2.0)_including standardization of reporting. Spinal Cord. 2017;55:759-64. 


\section{Affiliations}

\section{Kim D. Anderson $\mathbb{D}^{1,2} \cdot$ Edelle C. Field-Fote ${ }^{3,4,5} \cdot$ Fin Biering-Sørensen $\mathbb{D}^{6} \cdot$ Anne Bryden $^{7} \cdot$ Lisa A. Harvey $^{8} \cdot$ Linda Jones $^{9} \cdot$ M. J. Mulcahey ${ }^{10} \cdot$ Sara Mulroy $^{11,12} \cdot$ Vanessa K. Noonan $^{13,14} \cdot$ Marcel W. M. Post (iD $^{15}$. Rüdiger Rupp ${ }^{16}{ }^{16}$ Mary Schmidt-Read ${ }^{17}$}

1 MetroHealth Rehabilitation Institute, MetroHealth System, Cleveland, $\mathrm{OH}$, USA

2 Department of Physical Medicine and Rehabilitation, Case Western Reserve University, Cleveland, OH, USA

3 Crawford Research Institute, Shepherd Center, Atlanta, GA, USA

4 Division of Physical Therapy, Emory University School of Medicine, Atlanta, GA, USA

5 Program in Applied Physiology, School of Biological Sciences, Georgia Institute of Technology, Atlanta, GA, USA

6 Clinic for Spinal Cord Injuries, Neuroscience Centre, Rigshospitalet, University of Copenhagen, Copenhagen, Denmark

7 Institute for Functional Restoration, Case Western Reserve University, Cleveland, OH, USA

8 John Walsh Centre for Rehabilitation Research, Sydney Medical School, University of Sydney, Sydney, NSW, Australia

9 Anschutz Medical, University of Colorado, Denver, CO, USA
10 Department of Occupational Therapy, Center for Outcomes and Measurement, Jefferson College of Rehabilitation Sciences, Jefferson (Philadelphia University+Thomas Jefferson University), Philadelphia, PA, USA

11 Pathokinesiology Laboratory, Rancho Los Amigos National Rehabilitation Center, Downey, CA, USA

12 Rehabilitation Engineering Program, Rancho Los Amigos National Rehabilitation Center, Downey, CA, USA

13 Praxis Spinal Cord Institute, Vancouver, BC, Canada

14 Blusson Spinal Cord Centre, Vancouver, BC, Canada

15 Center of Excellence for Rehabilitation Medicine, Brain Center Rudolf Magnus, University Utrecht and De Hoogstraat Rehabilitation, Utrecht, Netherlands

16 Spinal Cord Injury Center, Heidelberg University Hospital, Heidelberg, Germany

17 Magee Rehabilitation Hospital, Jefferson Health, Philadelphia, PA, USA 